\title{
MODELING OF ECONOMIC SYSTEMS. GAME APPROACH
}

\section{Medvediev M. H.}

\section{General Model}

Let as suppose that a number of players participate in a game where they follow certain rules. The win that everyone gets as a result depends on their own actions as well as the actions of other players. If we consider this game in terms of its logical characteristics, abstracting from its social content, we will notice a clear similarity with the situations we discussed. The players are our participants in the economic process, the rules of the game - our setting or physical or institutional constraints, the winnings our usefulness or income. That is why the general concept of game theory is well applicable to the study of economic sphere ${ }^{1}$.

We denote each player or participant by the index $r$ or $s(r, s=1,2, \ldots, n)$. Actions $r$ can be represented in an adequate mathematical way, which in the general case is a vector $a_{r}$ in some space. Rules or restrictions require that $a_{r}$ should belong to some predetermined set $A_{r}$.

$$
a_{r} \in A_{r}, r=1,2, \ldots, n
$$

The player who wins the prize $r$, is a numerical function of the actions made between all participants:

$$
W_{r}\left(a_{1}, a_{2}, \ldots, a_{n}\right) \text {. }
$$

This presentation of the game is rather conditional. But it does not suggest that the game consists of one move and all players act at the same time. In fact, $a_{r}$ should be interpreted as a strategy that determines the actions of player $r$ at each move in all situations in which he/she may find him/herself as a the result of other players' actions. Let us suppose, for example, that a two-player game (A and B) consists of three moves, with the first one making the first and third moves and the second making the second move. Let us suppose that B has only two possible moves, denote them respectively by 1 and 2; player A knows in

\footnotetext{
${ }^{1}$ Malenvo E. Lectures on Microeconomic Analysis. Moskow: Science, 1985, 392 p.
} 
the third move which choice is made by player B. Actions $a_{r}$ of player A will thus have three components: what $\mathrm{A}$ does in the first move; what he does in the third move if $\mathrm{B}$ chose 1 , and what he does on the third move if $\mathrm{B}$ chose 2 . In games, even if not very difficult, $a_{r}$ a component has obviously a very large number: presenting a game with $A_{r}$ and $W_{r}$ can be very complicated. But this is not a barrier to abstract and general exploration. In setting such a logical structure, the problem of game theory is to determine what actions are taken or should be taken by players if each of them knows not only their own multitude and their own function but also the multitude $A_{s}$ and the win functions $W_{s}$, of other players.

It should be noted that the knowledge of $A_{s}$ and $W_{s}$ envisaged by all participants may prove to be very limiting for the application of game theory to the study of economic phenomena. It contains the natural assumption that the number of participants is small and each of them can effortlessly learn about the conditions of activity of each other participant. However, it is clear that this assumption makes game theory inadequate for the consideration of all the issues that arise from the need to organize information sharing in communities with large numbers of participants.

Game theory, if it were able to provide a general solution to the problem, could form the basis of a broad field of microeconomic theory. In all game theory, the difference between the presence and absence of cooperation between participants is essential both for formalization and for exploring the applicability of one or another of its variants.

In the formal examination, the above mentioned difficulties relate to the choice of general concepts, which allows to describe the result of cooperation between the participants. This choice is not easy. But it does not cause difficulties if the cooperation is removed. The concept of noncooperative equilibrium, which is also called the Nash equilibrium, is natural and can be applied to quite a variety of situations. Such an equilibrium $E_{0}$ is a possible state, that is, a set of certain values of $a_{1}{ }^{0}, a_{2}{ }^{0}, \ldots, a_{n}{ }^{0}$ vectors $a_{1}, a_{2}, \ldots, a_{n}$ belonging to a set $A_{r}$ that

$$
\begin{aligned}
& W_{r}\left(a^{0}{ }_{1}, \ldots, a_{r-1}^{0}, a_{r}, a^{0}{ }_{r+1}, \ldots, a_{r}^{0}, a^{0}{ }_{r+1}, \ldots, a^{0}{ }_{n}\right) \leq \\
& \leq W_{r}\left(a_{1}{ }^{0}, \ldots, a_{r-1}^{0}, a_{r}^{0}, a_{r+1}^{0}, \ldots, a_{r}^{0}, a^{0}{ }_{r+1}, \ldots, a_{n}^{0}\right)
\end{aligned}
$$


for all $a_{r}$ is $A_{r}$ and for all $\mathrm{r}$. In other words, $E_{0}$ is a non-cooperative equilibrium if neither participant is interested in changing its actions and if he/she considers the actions of others as set ones.

As we can see in the two examples, the non-cooperative equilibrium is not very plausible for the large number of cases in which the number of participants is small, because each of them is aware that his/her decisions affect the decisions of his/her partners. On the contrary, the case where there are many participants and each of them is insignificant and poorly aware of the other's capabilities, is more in line with the non-cooperative equilibrium in which the participants' awareness requirements are low.

Therefore, the structure of the participant community is essential when choosing between these two basic assumptions, but it is not just that.

The nature of the relationship between participants (partners and adversaries, suppliers and clients, managers and their employees, etc.) also influences the degree of cooperation that is established between them. and duopoly. To begin the consideration of the application of game theory having imperfect competition, let us first consider a bilateral monopoly and a duopoly.

Let us note that most of the models studied using economic theory are complications of the general game theory model: the set of $A_{r}$ possible actions of a participant $r$ is initially not completely specified, but partly depends on the actions of other participants, i.e

$$
a_{r} \in A_{r}\left(a_{1}, \ldots, a_{r-1}, a_{r+1}, \ldots, a_{n}\right), r=1,2, \ldots, n .
$$

However, this complication does not essentially relate to the definition of basic concepts, such as the Nash equilibrium. (Of course, this implies that $n$ conditions (38) are not mutually contradictory).

\section{Bilateral Monopoly}

Bilateral monopoly is a situation where one consumer and one supplier act on the market of some goods.

We believe that the first is such a good, and in the markets of other goods there is perfect competition. We also believe that both the consumer and the supplier are enterprises, and the good 1 is the intermediate, that is, the products of the first enterprise and the resource of the second. For both the supplier and the consumer the prices of other goods are set. Both partners must agree on the price of $p_{1}$ and the amount of good 1 that is exchanged. 
Let us suppose that $C_{1}\left(y_{1}\right)$ is the cost of production of the supplier enterprise, $R_{2}\left(y_{1}\right)-p_{1} y_{1}$ is the profit received by the consumer enterprise as a result of the use of $y_{1}$.

The profits of both participants are equal to

$$
W_{1}=p_{1} y_{1}-C_{1}\left(y_{1}\right), W_{2}=R_{2}\left(y_{1}\right)-p_{1} y_{1} .
$$

Let us suppose that $C_{1}$ and $R_{2}$ are twice differentiated functions, $C^{\prime \prime}{ }_{1}>0, R^{\prime \prime}{ }_{2}<0$.

To determine the payoff functions, as it is customary in game theory, we need to clarify the actions $a_{1}$ and $a_{2}$ of both entrepreneurs and the respective areas $A_{1}$ and $A_{2}$. It is possible to make various models, which are different variants of a bilateral monopoly and contain a specific definition of a pair $\left(p_{1}, p_{2}\right)$ as a function of performed actions $\left(a_{1}, a_{2}\right)$. We believe that the first enterprise $\mathrm{A}$ determines the price $p_{1}$, and the second enterprise $\mathrm{B}-$ the amount that it will buy, i. e $y_{1}$. Areas $A_{1}$ and $A_{2}$ are thus defined for $p_{1} \geq 0$ and $y_{1} \geq 0$ respectively.

We find out what the non-cooperative equilibrium is. Enterprise B, if it considers the price $p_{1}$ as a given value, behaves as if the market for this good was competitive. It selects $y_{1}$ that

$$
R_{2}^{\prime}\left(y_{1}\right)=p_{1} \text { or leaves } y_{1}=0 \text { if } R_{2}^{\prime}(0)<p_{1}
$$

The first enterprise, if it considers $y_{1}$ as a given value, is interested in setting perhaps a higher price $p_{1}$ (infinitely large if the area is $A_{1}$ unlimited), except when $y_{1}=0$, i.e when $p_{1}$ can be selected by anyone. Strictly speaking, the only possible non-cooperative equilibrium is $y_{1}=0$ and $p_{1} \geq R_{2}{ }^{\prime}(0)$, which results in zero output of the good under consideration. Obviously, Enterprise A, when choosing $p_{1}$, cannot ignore the impact that this choice will have on enterprise B. It should not set a very high price that would lead to the disappearance of demand, but could maximize its profit, given that its partner sets the $y_{1}$ and according to (40).

In this case, it will act as a monopolist, the demand for products is determined by this equation Simple calculations show that in this case it will produce pure products in the quantity $y_{1}{ }^{*}$, which is the solution of the equation

$$
C_{1}{ }^{\prime}\left(y_{1}\right)-y_{1} R_{2}{ }^{\prime \prime}\left(y_{1}\right)=R_{2}{ }^{\prime}\left(y_{1}\right)
$$

and sell it for $\boldsymbol{p}_{\mathbf{1}}{ }^{*}=\boldsymbol{R}_{\mathbf{2}}{ }^{\prime}\left(\boldsymbol{y}_{\mathbf{1}}{ }^{*}\right)$. But the company $B$ can not satisfy equation (40) because it knows that $A$ is the only partner. It may, for example, refuse to purchase for the price $\boldsymbol{p}_{1}{ }^{*}$ the whole quantity of 
products $\boldsymbol{y}_{1}{ }^{*}$, having the right to believe that such a position will force $A$ to agree to a price reduction. Before defining its actions, every enterprise is interested in discovering a rule of behavior that another enterprise will follow. It can do this by putting itself in the place of a partner and determining the most appropriate rule for him.

Thus, both enterprises should understand immediately or after a mutual «probe» that it is advantageous for them to reach an explicit or implicit agreement that would be acceptable to both of them. It is indifferent that the first sets $\boldsymbol{p}_{1}$, and the second $-\boldsymbol{y}_{\mathbf{1}}$, as they thus act together to determine the acceptable combination $\left(\boldsymbol{p}_{\mathbf{1}}{ }^{\mathbf{0}}, \boldsymbol{y}_{\mathbf{1}}{ }^{\mathbf{0}}\right)$

This combination must satisfy the following conditions:

1) the profit $\boldsymbol{W}_{\mathbf{1}}$ is at least equal to $\boldsymbol{C}_{\mathbf{1}}(\mathbf{0})$, otherwise case $A$ is not interested in exchange with $\mathrm{B}$;

2) $\boldsymbol{W}_{\mathbf{2}}$ is at least equal to $\boldsymbol{R}_{\mathbf{2}}(\mathbf{0})$;

3) the combination maximizes $\boldsymbol{W}_{\mathbf{1}}$ provided that $\boldsymbol{W}_{\mathbf{2}}$ preserves the value $\boldsymbol{W}_{\mathbf{2}}{ }^{\mathbf{0}}$, otherwise $A$ could offer $B$ a more acceptable combination for itself, which would also remain good for B;

4) the combination maximizes $\boldsymbol{W}_{2}$ provided that $\boldsymbol{W}_{1}$ preserves the value $\boldsymbol{W}_{\mathbf{1}}{ }^{\mathbf{0}}$.

To clarify the above mentioned we find out what follows from condition 3 ). If we put $\boldsymbol{y}_{\mathbf{1}} \neq \mathbf{0}$, then from 3 ) it follows that such a number $\lambda$ exists that the derivatives of the expression

$\left[p_{1} y_{1}-C_{1}\left(y_{1}\right)\right]+\lambda\left[R_{2}\left(y_{1}\right)-p_{1} y_{1}\right]$ of $p_{1}$ and $y_{1}$ simultaneously turn into zero. A derivative of $\boldsymbol{p}_{\mathbf{1}}$ equals to zero when $\boldsymbol{\lambda}=\mathbf{0}$. Since the derivative of is zero, we have an equation

$$
C_{1}{ }^{\prime}\left(y_{1}{ }^{0}\right)=R_{2}{ }^{\prime}\left(y_{1}{ }^{0}\right),
$$

which defines $\boldsymbol{y}_{\mathbf{1}}{ }^{\mathbf{0}}$ in a unique way as $\boldsymbol{C}_{\mathbf{1}}{ }^{\prime}$ increases and $R_{2}{ }^{\prime}$ decreases.

The study of condition 4) leads, obviously, to the same result. Conditions 1) and 2) thus determine the interval to which the price should belong $\boldsymbol{p}_{\mathbf{1}} \mathbf{0}$ :

$$
C_{1}\left(y_{1}{ }^{0}\right)-C_{1}(0) / y_{1}{ }^{0} \leq{p_{1}}^{0} \leq R_{2}\left({y_{1}}^{0}\right)-R_{2}(0) / y_{1}{ }^{0}
$$

This means that all combinations $\left(\boldsymbol{p}_{\mathbf{1}}{ }^{\mathbf{0}}, \boldsymbol{y}_{\mathbf{1}}{ }^{\mathbf{0}}\right)$ that allow both parties to come to an agreement contain the same amount of products, and the price must be in the interval (42). Thus, there are many similar combinations. We will assume that this set is the kernel of bilateral monopoly. 
Let us show the set in the graph, on the abscissa axis of which $\boldsymbol{y}_{\mathbf{1}}$, is laid, and on the y-axis $-\boldsymbol{p}_{\mathbf{1}}$ (Fig. 1). The dashed curve corresponds to combinations for which $\boldsymbol{W}_{1}$ or $\boldsymbol{W}_{2}$ takes the same set value. Curves $W_{1}=$ constand $W_{2}=$ const touch each other at the points of vertices with abscissa $\boldsymbol{y}_{\mathbf{1}}{ }^{\mathbf{0}}$. The kernel is represented by the interval $R S$ of this vertical, which is located between the two curves passing through the origin.

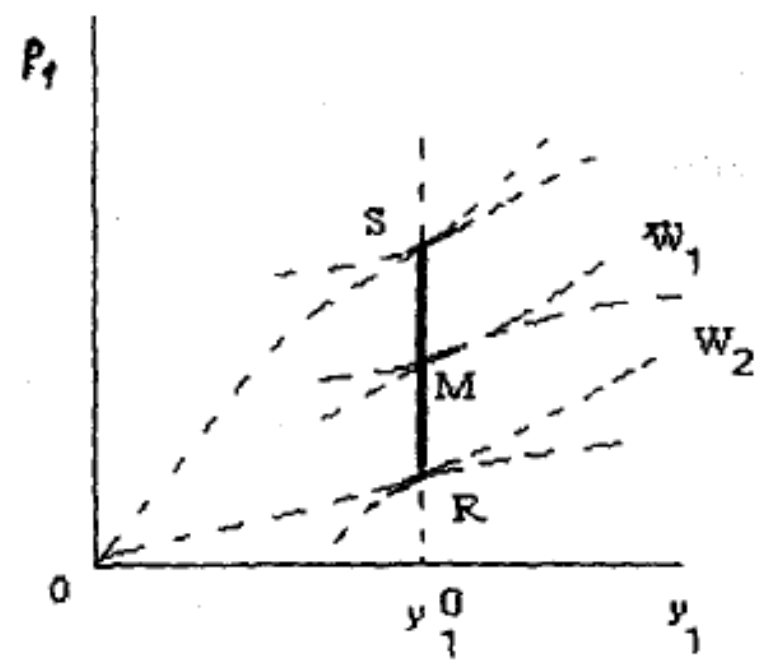

Fig. 1

How can $p_{1}$ be determined inside the interval (42)? Enterprise $A$ is interested in choosing the largest price, and enterprise $B$ interested in choosing the lowest price. Inside the core, the interests of both partners are completely opposite. Therefore, they believe that the final combination chosen depends on the relative power of both partners. Each may be threatened with refusal to comply with the agreement and thus persuade the other to fulfill their requirements. However, none of the partners can substantiate their threats by being able to make a big profit alone by refusing to cooperate altogether. Threats are only effective if an agreement is eventually obtained.

In view of the above mentioned, we can draw the following conclusions.

1. The non-cooperative equilibrium is not a productive competition of bilateral monopoly.

2. Partners are interested in negotiating with each other and executing one of the core-owned combinations. 
3. Using threats as a means of achieving a particularly advantageous combination has the risk of breaking the agreement, which will eventually lead to an out-of-core combination.

\section{Duopoly}

Let us consider the theory of duopoly, which is a market maintained by two manufacturers, in which demand is determined by numerous but small-size consumers. Economic theory gives an idea of this situation, assuming that each unit of good under consideration is exchanged at the same price and demand is competitive in the sense that the total quantity of sold products depends only on its price (and therefore it makes no sense to include for the consideration the individual consumer strategies). For convenience, we consider that this is a good 1 market and that the demand function is decreasing and can be written down

$$
p_{1}=\pi\left(y_{1}\right)
$$

as for monopoly. The total number of pure products $\boldsymbol{y}_{\mathbf{1}}$ is produced by enterprises 1 and 2 , each of which produces respectively pure products in the quantities $\boldsymbol{y}_{11}$ and $\boldsymbol{y}_{21}$.

For the study of the duopoly, let us suppose that the prices $\boldsymbol{p}_{2}, \boldsymbol{p}_{3}, \ldots, \boldsymbol{p}_{\mathbf{L}}$ of other goods are determined, for example in competitive markets, and do not depend on $\boldsymbol{p}_{\mathbf{1}}$ and $\boldsymbol{y}_{\mathbf{1}}$. Strictly speaking, this is possible only when the good 1 is relatively insignificant and thus the demand of enterprises 1 and 2 in the markets of other goods can be neglected. The function $\boldsymbol{\pi}$, obviously, depends on the values $\boldsymbol{p}_{2}, \boldsymbol{p}_{3}, \ldots, \boldsymbol{p}_{\mathbf{L}}$ as parameters. Let us denote the cost functions of enterprises 1 and 2 by $\boldsymbol{C}_{\mathbf{1}}\left(\boldsymbol{y}_{11}\right)$ and $\boldsymbol{C}_{2}\left(\boldsymbol{y}_{21}\right)$. The corresponding profits will be

$$
\begin{aligned}
& W_{1}\left(y_{11}, y_{12}\right)=y_{11} \pi\left(y_{11}+y_{21}\right)-C_{1}\left(y_{11}\right) \\
& W_{2}\left(y_{11}, y_{21}\right)=y_{22} \pi\left(y_{11}+y_{21}\right)-C_{2}\left(y_{21}\right)
\end{aligned}
$$

Since the quantities of pure products $\boldsymbol{y}_{\mathbf{1 1}}$ and $\boldsymbol{y}_{\mathbf{2 1}}$ are variable, they reflect the behavior of both enterprises, $\boldsymbol{W}_{\mathbf{1}}$ and $\boldsymbol{W}_{\mathbf{2}}$ are their profit functions respectively.

A. Cournot, who first investigated the theory of duopoly, proposed as a solution the non-cooperative equilibrium, which, when applied to the duopoly, is called the Cournot equilibrium. This solution assumes that each enterprise passively observes the other enterprise and accepts its choice as 
a given one, and then makes its own choice so as to maximize its profit. The equilibrium in this case is determined by the pair $\left(\boldsymbol{y}_{\mathbf{1 1}_{1}}, \boldsymbol{y}^{\mathbf{0}}{ }_{\mathbf{2 1}}\right)$ that $\boldsymbol{y}_{\mathbf{1 1}}$ maximizes $\boldsymbol{W}_{\mathbf{1}}\left(\boldsymbol{y}_{\mathbf{1 1}_{1}}^{\mathbf{0}}, \boldsymbol{y}^{\mathbf{0}}{ }_{\mathbf{2 1}}\right)$, which is considered as a function $\boldsymbol{y}_{\mathbf{1 1}}$, and $\boldsymbol{y}_{\mathbf{2 1}}$ maximizes $\boldsymbol{W}_{\mathbf{2}}\left(\boldsymbol{y}_{{ }_{11}}^{\mathbf{0}}, \boldsymbol{y}^{\mathbf{0}}{ }_{21}\right)$, which is considered as a function $\boldsymbol{y}_{\mathbf{2 1}}$.

However, in this situation, it is even less obvious than under a bilateral monopoly that enterprises occupy a similar passive position (Fig. 2).

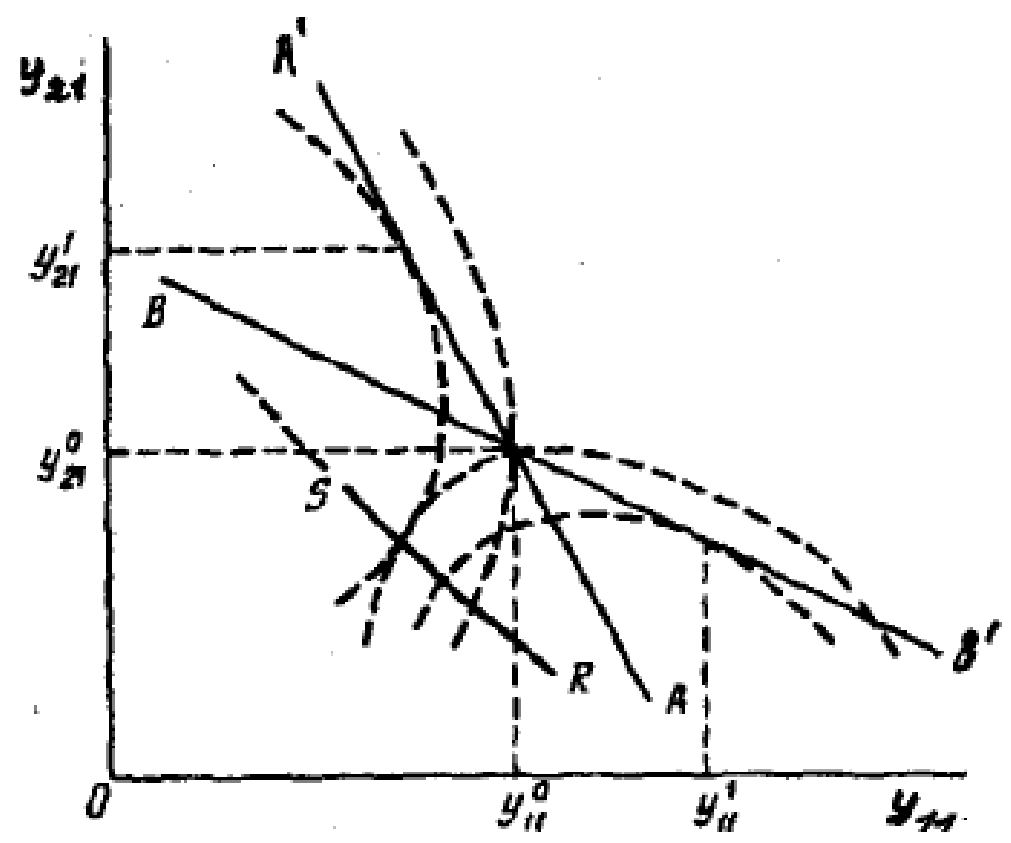

Fig. 2

The curves bent down are lines of level $\boldsymbol{W}_{\mathbf{1}}=$ const; curves, curved to the left are the lines $W_{2}=$ const. Curve $A A$ ' is the geometric location of the points of the lines of level $\boldsymbol{W}_{\mathbf{1}}=$ const, which have the largest ordinate. It determines for each $\boldsymbol{y}_{\mathbf{2 1}}$ the choice of enterprise 1 if it occupies a passive position. In fact, the profit $\boldsymbol{W}_{\mathbf{1}}$ obviously increases when moving down along the vertical and, thus, on the horizontal $\left(\boldsymbol{y}_{\mathbf{2 1}}\right.$ set) enterprise 1 is interested in choosing the coordinate of the point at which this horizontal touches the lines of the level $\boldsymbol{W}_{\mathbf{1}}=$ const.

Similarly, the curve $\boldsymbol{B} \boldsymbol{B}^{\prime}$, that connects the most right points of the lines of the level $\boldsymbol{W}_{\mathbf{2}}=$ const, determines the behavior of enterprise 2 when it takes a passive position.

Thus, the Cournot equilibrium is the point of intersection of curves $\boldsymbol{A} \boldsymbol{A}^{\prime}$ and $\boldsymbol{B} \boldsymbol{B}^{\prime}$, let it be $\left(\boldsymbol{y}_{\mathbf{1 1}}^{\mathbf{0}}, \boldsymbol{y}^{\mathbf{0}}{ }_{\mathbf{2 1}}\right)$. However, it is assumed that enterprise 
1 knows not only its function $W_{1}$ but also the function $W_{2}$ of its competitor. It can then determine the curve $\boldsymbol{B} \boldsymbol{B}^{\prime}$, that characterizes the behavior of enterprise 2 if it takes a passive position. In this case, enterprise 1 is interested in choosing the point on the curve $\boldsymbol{B} \boldsymbol{B}^{\prime}$ at which it touches the curve $\boldsymbol{W}_{\mathbf{1}}=$ const, that is, in the production of quantity $\left(\boldsymbol{y}_{11}\right.$, which is significantly higher in our case $\boldsymbol{y}^{\mathbf{0}}{ }_{\mathbf{1 1}}$. It is likely that enterprise 1 is aware that it can make more profit than with the Cournot equilibrium. It will then select, for example, production $\boldsymbol{y}_{\mathbf{1 1}_{11}}$. But the same considerations are applied to enterprise 2, which is interested in choosing production $\boldsymbol{y}_{\mathbf{2 1}}^{\prime}$ if it states the passive position of its competitor. At the same time, choosing a pair $\left(\boldsymbol{y}_{11}^{\prime}, \boldsymbol{y}_{\mathbf{2 1}}^{\prime}\right)$ means a profit for both enterprises that is much less than provided by the Cournotes equilibrium.

As with a bilateral monopoly, each participant, while accepting the situation of the other, must sooner or later come to an explicit or implicit agreement with him, since only in this case one can avoid a struggle that harms both competitors, on the assumption that neither of them believes that it can oust another from the market. An agreement is possible in such pairs $\left(\boldsymbol{y}_{11}^{\prime}, \boldsymbol{y}_{21}^{\prime}\right)$ when, on the one hand, each enterprise makes a profit at least equal to what it would gain by withdrawing from the market and which on the other hand, maximizes the profit of one enterprise at a given value of the other enterprise's profits. These pairs are depicted in Fig. 7 by the points of the curvilinear segment $R S$, belonging to the curve connecting the points of contact of the lines of levels $\boldsymbol{W}_{1}=$ constand $\boldsymbol{W}_{2}=$ const, where the point $R$ is located on the curve $\boldsymbol{W}_{\mathbf{1}}=-\boldsymbol{C}_{\mathbf{1}}(\mathbf{0})$ and the point $S$ - on the curve $\boldsymbol{W}_{2}=-\boldsymbol{C}_{2}(\mathbf{0})$

Як and in a bilateral monopoly, the set of pairs depicted by the points $R S$, can be called a core. Inside the core, the position of the pair $\left(\boldsymbol{y}_{11}, \boldsymbol{y}_{12}\right)$ seems uncertain at first. Each of the two enterprise can try to achieve a particularly advantageous combination for itself, threatening to refuse to fulfill the agreement. But this position is only beneficial if the threat is not fulfilled. The implementation of the combination within the kernel is specified by the agreement between the two enterprises, which, of course, will not behave as the monopolist would have done in their place. The monopoly is trying to increase the total profit $\boldsymbol{W}_{\mathbf{1}}+\boldsymbol{W}_{2}$, , which would usually lead to an unambiguous determination of the pair $\left(\boldsymbol{y}_{\mathbf{1 1}_{1}}, \boldsymbol{y}^{*}{ }_{\mathbf{2 1}}\right)$ inside the core. 


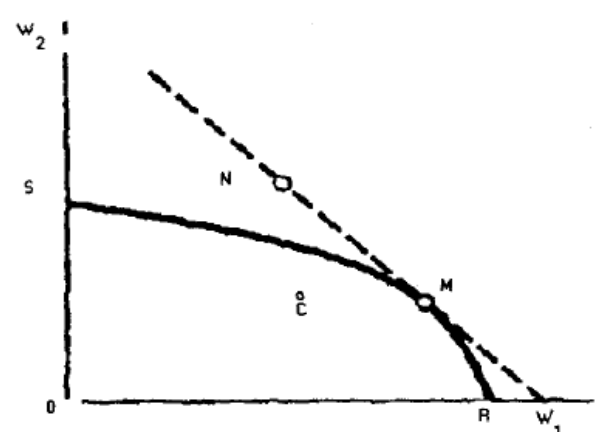

Fig. 3

The difference in their behavior can be traced in Fig. 3, in which the values of profits $W_{1}$ and $W_{2}$ are laid along the abscissa and the ordinate. The kernel is depicted by the curve $R S$, which limits the top and right sets of combinations $\left(W_{1} W_{2}\right)$, which follow from all choices of values $\boldsymbol{y}_{11}$ and $\boldsymbol{y}_{\mathbf{2 1}}$. (The Cournot equilibrium is represented by a point $\mathrm{C}$ inside $R S$.) The sum $W_{1}+W_{2}$ is maximum for the combination $M$, at which tangent to the curve $R S$ is parallel to the bisectric line. Point $M$ does not necessarily satisfy the two enterprises equally. The enterprise may not agree with choosing this point, which hopes to achieve a more profitable point for it on $R S$. However, we must remember that if there is a complete agreement between the two enterprises, then they can implement any point on tangent to $R S$ at point $M$, e.g $N$.To do this, it is sufficient for them to agree to a direct payment of one enterprise to another. In our case, shown in Fig. 3, one enterprise must pay another the sum equal to the projection length of the segment NMon the corresponding coordinate axis. In the case of a full agreement, both enterprises behave as one monopolist, and the only disputable issue between them is the division of total profit,, that is, a decision on a side payment, which one party must provide to the other. In the process of discussing this, everyone can obviously exploit threats, at the risk of breaking the deal.

The two cases considered give us the right to draw several conclusions.

1. The implementation of non-cooperative equilibrium is, as we see, impossible.

2. If there are hidden or explicit agreements, then it is possible to make judgments based on them and without paying attention to the actions of the participants themselves (only combinations of winnings that are possible as decisions of the game are important). 


\section{Trade when concluding transactions}

Let as suppose that a certain number of players participate in a game where they follow certain rules. The gain that each of them will receive as a result depends on his or her own actions and the actions of the other players. Before determining their actions, each enterprise is naturally interested in discovering a rule of behavior that will be imitated by another enterprise. It can do this by putting itself in the place of a partner and determining the most appropriate rule for him. That is, as noted, a kernel is formed, within which a compromise solution for both parties must be chosen if they are genuinely interested in reaching an agreement. However, the kernel contains many elements, and there are doubts as to which of them should ultimately be selected. It is quite appropriate to try to find a deterministic solution in the absence of additional circumstances, such as a bilateral monopoly (a situation where one consumer and one supply stand in the market of some goods, a duopoly (the market is maintained by two producers, whose demand is determined by numerous but insignificant consumers). and some others. In fact, any logical analysis of the complications that occur in each case can lead to the same problem - the multiplicity of possible outcomes. It is advisable to try to deduce the principles for finding such a solution. It is about principles, that is, finding a general rule for a category of situations.

The problem of so-called trade defines the scope of this study. They are easy to define. The vector $\boldsymbol{\omega}$ of winnings $\boldsymbol{\omega}_{1}$ and $\boldsymbol{\omega}_{2}$ of both participants must belong to the set $P$. It is known that it will take the value $\boldsymbol{v}$ (which belongs, obviously, to $P$ ), unless the parties reach an agreement. What vector $\boldsymbol{\omega}^{*}$ of $P$ should be agreed with? The general answer is to find out how $\boldsymbol{\omega}^{*}$ depends on $P$ and $\boldsymbol{v}$; this (solvable) function allows you to get a solution

$$
\omega^{*}=\mu(v, P)
$$

the value of which is defined on the set $P$.

In order to investigate the properties that the function $\boldsymbol{\mu}(\boldsymbol{v}, \boldsymbol{P})$ should possess and to consider its capabilities arising from these features, we have to accept several common axioms.

A comprehensive answer to this question was given by J. Nesh, who found it necessary to accept the following four axioms.

A1. The solution must be the Pareto optimal,in other words $\boldsymbol{\mu}(\boldsymbol{v}, \boldsymbol{P})$ should be located on the boundary of $P$ on the upper right. 
A2. The solution must be individually rational in the sense that each participant should receive a win no less than that which he or she would have received in the absence of the agreement, i.e p $\boldsymbol{\mu}(\boldsymbol{v}, \boldsymbol{P}) \geq \boldsymbol{v}$.

A3. The decision should not be changed if $P$ is replaced by a subset Qcontained in $P$ and containing $\boldsymbol{\mu}(\boldsymbol{v}, \boldsymbol{P})$

A4. If there are two linear increasing functions $\boldsymbol{\varphi}_{1}$ and $\boldsymbol{\varphi}_{2}$ that the conditions

$$
v_{i}^{2}=\varphi_{i}\left(v_{i}^{1}\right), i=1,2,
$$

and $\in \boldsymbol{P}^{\mathbf{1}}$, when and only when

$$
\varphi(\omega) \in P^{2},
$$

are carried out, then the decisions $\left(\boldsymbol{v}^{\mathbf{1}}, \boldsymbol{P}^{\mathbf{1}}\right)$ and $\left(\boldsymbol{v}^{\mathbf{2}}, \boldsymbol{P}^{\mathbf{2}}\right)$ must be the same in the sense that $\boldsymbol{\mu}\left(\boldsymbol{v}^{\mathbf{2}}, \boldsymbol{P}^{\mathbf{2}}\right)=\boldsymbol{\varphi}\left[\boldsymbol{\mu}\left(\boldsymbol{v}^{\mathbf{1}}, \boldsymbol{P}^{\mathbf{1}}\right)\right]$. Using some lowboundary conditions for $(\boldsymbol{v}, P)$, Nash showed that there is the only one function that satisfies the axioms $A 1-A 4$. More precisely, $\boldsymbol{\mu}(\boldsymbol{v}, \boldsymbol{P})$ is a vector that maximizes in $P$ the product $\left(\boldsymbol{\mu}_{\mathbf{1}}-\boldsymbol{v}_{\mathbf{1}}\right) \cdot\left(\boldsymbol{\mu}_{\mathbf{2}}-\boldsymbol{v}_{\mathbf{2}}\right)$ of additional wins that both participants receive from their collaboration.

\section{Coalition and Decisions}

The distribution is the $n$-dimensional vector $\left(\boldsymbol{\omega}_{1}, \boldsymbol{\omega}_{2}, \ldots, \boldsymbol{\omega}_{r}, \ldots, \boldsymbol{\omega}_{n}\right)$, the components of which are players' winnings prior to the end of the game. Distribution is possible if there is a multiple of possible distributions $n$ of players, which allows to make winnings corresponding to such distribution. Most often, for the participant $r$ there is a minimum value $\boldsymbol{v}_{\boldsymbol{r}}$ of winnings, which he can provide for himself regardless of the actions of other players. For example, in an exchange economy, it will be the usefulness $\boldsymbol{S}_{\boldsymbol{r}}\left(\boldsymbol{\omega}_{\boldsymbol{r}}\right)$ that he/she will receive if abandons other exchanges.

The distribution $\left(\boldsymbol{\omega}_{1}, \boldsymbol{\omega}_{2}, \ldots, \boldsymbol{\omega}_{r}, \ldots, \boldsymbol{\omega}_{n}\right)$ is considered to be individually rational if $\boldsymbol{\omega}_{\boldsymbol{r}} \geq \boldsymbol{v}_{\boldsymbol{r}}$ for all $r$. In fact, it is a priori possible to remove from consideration a result in which some participant does not receive the minimal win that he can provide himself with. It is also believed that the distribution $\omega$ is rejected by or is blocked by a player $i$ if $\boldsymbol{\omega}_{\boldsymbol{i}}<\boldsymbol{v}_{\boldsymbol{i}}$. . Therefore, individually rational distribution is not blocked by any participant.

By definition the coalition a subset $\mathrm{C}$ of the set $I$ is meant, consisting of players: $\boldsymbol{I}=\{\mathbf{1}, \mathbf{2}, \ldots, \boldsymbol{n}\}$. In a theoretical study, it is convenient to 
preserve the term «coalition» to denote both the entire set Iand a subset consisting of one player $r$, for example $\{r\}$. The possibility of coalitions influences the outcome of the game, since only one coalition can achieve some result, or a particular coalition may block the implementation of another result. To investigate this issue, we introduce a simple formalization.

Distribution $\left(\boldsymbol{\omega}_{1}, \boldsymbol{\omega}_{2}, \ldots, \boldsymbol{\omega}_{\boldsymbol{r}}, \ldots, \boldsymbol{\omega}_{\boldsymbol{n}}\right)$ is called possible for coalition $C$, if $C$ can provide its members with winnings $\boldsymbol{\omega}_{\boldsymbol{r}}$ (for $\boldsymbol{r} \in \boldsymbol{C}$ ), whatever actions are made by players which are not in $C$. Coalition canblock making some distribution if it can provide its members with more winnings than that distribution. Therefore, a formal definition can be given. Coalition $C$ blocks distribution $\left(\boldsymbol{\omega}_{\mathbf{1}}{ }^{\mathbf{0}}, \boldsymbol{\omega}_{\mathbf{2}}{ }^{\mathbf{0}}, \ldots, \boldsymbol{\omega}_{\boldsymbol{r}}{ }^{\mathbf{1}}, \ldots, \boldsymbol{\omega}_{\boldsymbol{n}}{ }^{\mathbf{0}}\right)$ if there is possible a distribution $\left(\boldsymbol{\omega}_{1}{ }^{\prime}, \boldsymbol{\omega}_{2}{ }^{\prime}, \ldots, \boldsymbol{\omega}_{\boldsymbol{r}}{ }^{\prime}, \ldots, \boldsymbol{\omega}_{\boldsymbol{n}}{ }^{\prime}\right)$ that $\boldsymbol{\omega}_{\boldsymbol{r}}{ }^{\prime} \geq \boldsymbol{\omega}_{\boldsymbol{r}}{ }^{\mathbf{0}}$ for each player $r$ from $C$ and $\boldsymbol{\omega}_{\boldsymbol{r}}{ }^{\mathbf{1}} \geq \boldsymbol{\omega}_{\boldsymbol{r}}{ }^{\mathbf{0}}$ for at least one player $r$ from $C$. As an example, let us consider a bilateral monopoly. Let the enterprise $A$ be a player 1 , and the enterprise $B$ be a player 2. Coalition $\{1\}$, consisting of single player 1 , blocks any distribution corresponding to player 1 winning less than $\boldsymbol{C}_{\mathbf{1}}(\mathbf{0})$; coalition $\{2\}$ blocks any distribution that matches player 2 winnings less than $\boldsymbol{R}_{\mathbf{2}}(\mathbf{0})$ coalition $\{1,2\}$ of two enterprises blocks any distribution that maximizes $\boldsymbol{W}_{\mathbf{1}}$ at a given value of $\boldsymbol{W}_{\mathbf{2}}$ or does not maximize $\boldsymbol{W}_{\mathbf{2}}$ at given value $\boldsymbol{W}_{\mathbf{1}}$. We state that the kernel thus consists of all possible combinations $\left(\boldsymbol{p}_{1}, \boldsymbol{y}_{\mathbf{1}}\right)$, corresponding to distributions that are not blocked by any coalition. Similar considerations can be made for the duopoly. This is the explanation of the following statement.

The kernel consists of the set of possible distributions that are not blocked by any of the coalitions.

The value of this statement lies in the idea that the game naturally leads to some kernel-owned distribution.

There are three situations where this is not the case.

1 . The use of threats by some players can break agreements and lead to outcomes adverse for all participants.

2. When the number of players is large enough, the information of each of them about the position of the other becomes often incomplete and the making of agreements, which a priori seems to be fruitful, may require long, costly negotiations. To reflect this, they talk about the costs of information and communication that make the participants sometimes content with non-core distributions. 
3. There are situations where the kernel is empty. This means that for every possible division, you can find a coalition that can block it.

This is explained by the fact that when considering cooperation and the clash of interests of many participants, game theory is not limited to a single concept of the kernel, which, however, is most commonly used in economic theory. The purpose of conceptual research in game theory is to find a good description of the likely outcome of the game. To do this, it would be enough to have a solution concept that satisfies three conditions: it gives an intuitively correct view; applicable to all or most cases; usually leads to a single solution of the problem. Three conditions cannot be satisfied at the same time. Thus, various existing theories are theoretical compromises.

We see that the kernel does not fully meet the last two conditions. It seems to fit well with the former. However, in some cases, the emergence of the blocking coalitions that are needed is doubtful as they involve reaching an agreement between the parties, the communication between which is difficult. This means that all blocking coalitions must be treated equally, regardless of their origin. in introducing some options to negotiate for players who obviously depend on the outcome of the game. In order to avoid the extreme consequences of this circumstance, we introduce the principle of finding solutions, which offers us to simultaneously consider all the coalitions in which each player can participate, and to introduce some opportunities to negotiate for players, on whom obviously the outcome of the game depends. This principle was introduced by Shapley and developed by him with M. Shubik. Regarding this principle, the chosen decision is thought to have a Shapley price, or just a price. Let us consider the contribution $\boldsymbol{g}_{\boldsymbol{r}}(\boldsymbol{C})$ that an individual $r$ contributes to a winning of a coalition $C$ if it becomes a part of it. For any Coalition $C$ that does not include $r$, this contribution is equal to the payoff that the considered coalition $\boldsymbol{C} \cup\{\boldsymbol{r}\}$ can receive, minus the payout that $\mathrm{C}$ can get. The definition of this contribution is simple when the winnings are transferable, i.e they can be transferred from one person to another so that the overall winnings retain value when using considerations close to those made for the trade problem).

The determination of the contribution $r$ in $\mathrm{C}$ can also be made in the case where the winnings are non-transferable. The Shapley price is defined as a distribution whose components are, accordingly, an average $\overline{\boldsymbol{g}}_{r}$ of 
values $\boldsymbol{g}_{\boldsymbol{r}} \boldsymbol{C}$ on the set of all coalitions $C$, that do not contain $r$. In the game each average determines the natural measure for the ability of the individual $r$ to reach an agreement - a measure that must be considered by others in such a way that, as a result of a general agreement, he can get a win equal to $\overline{\boldsymbol{g}}_{r}$ at the end of the game, thar determines the final division (the Shapley price). This concept is considered acceptable when considering some economic problems, and it is often an interesting alternative to the concept of the kernel, when the solution involves cooperation between participants. In each case, the question remains whether the most non-cooperative equilibrium is appropriate here. The larger the number of participants, the more complex the links between them are; the more problematic the possibility of a coalition is, the more plausible the realization of a non-cooperative equilibrium is. Conversely, a small number of participants, naturally interacting for a long time in recurring situations, are naturally cooperative.

\section{Arbitration and exchange between the parties}

After examining some special situations, let us return to the general economic models. We look for states that can be realized if the exchanges are made not under the laws of perfect competition. It is assumed that all forms of imperfect competition are a priori possible. Let us find out what states can be achieved.

Let us begin to study this problem without any preconceived idea, as Englishman Edgeworth did at the end of the XIX century. This consideration will help us better understand some aspects of equilibrium. We use the terminology adopted by M. Ale in exploring the same issues.

Let two consumer individuals $i$ and $\boldsymbol{\alpha}$ have the goods $\boldsymbol{x}_{\boldsymbol{i n}}$ and $\boldsymbol{x}_{\boldsymbol{\alpha} \boldsymbol{h}} \boldsymbol{h}=(\mathbf{1}, \mathbf{2}, \ldots, \boldsymbol{l})$ respectively. These are the numbers they originally owned $\left(\boldsymbol{\omega}_{\boldsymbol{i} h}\right.$ and $\left.\boldsymbol{\omega}_{\boldsymbol{\alpha} \boldsymbol{h}}\right)$ or as a result of exchanges. Let us suppose that the operation, which involves the exchange of goods, is beneficial for both. Denote by $\boldsymbol{z}_{\boldsymbol{h}}$ the amount of good $h$, which $i$ inferior to $\boldsymbol{\alpha}$ in such an operation, or by $\left(-\boldsymbol{z}_{\boldsymbol{h}}\right)$ the amount of this good, which $\boldsymbol{\alpha}$ inferior to $i$. Since the operation is beneficial for both, then $\boldsymbol{S}_{\mathbf{1}}\left(\boldsymbol{x}_{\boldsymbol{i}}-\boldsymbol{z}\right)>\boldsymbol{S}_{\boldsymbol{i}}\left(\boldsymbol{x}_{\boldsymbol{i}}\right)$, $\boldsymbol{S}_{\boldsymbol{\alpha}}\left(\boldsymbol{x}_{\boldsymbol{\alpha}}+\boldsymbol{z}\right)>\boldsymbol{S}_{\boldsymbol{\alpha}} \boldsymbol{x}_{\boldsymbol{\alpha}}$, where $S$ denotes utility. The possibility of such an operation may be unknown either $i$ or $\alpha$. In doing so, any third party that becomes an intermediary in carrying out this operation will be able to derive some benefit for themselves. In fact, because of the continuity $\boldsymbol{S}_{\boldsymbol{i}}$, there is a 
non-zero vector $\amalg$ with indefeasible components such as $\boldsymbol{S}_{\mathbf{1}}\left(\boldsymbol{x}_{\boldsymbol{i}}-\boldsymbol{z}-\boldsymbol{\omega}\right)>$ $>\boldsymbol{S}_{\boldsymbol{i}}\left(\boldsymbol{x}_{\boldsymbol{i}}\right)$. All three participants will find a exchange profitable. in which the quantities of benefits $h$ will change to $-\left(z_{h}+\omega_{h}\right)$ for $i$, to $z_{h}$ for $\alpha$ and to $\boldsymbol{\omega}_{\boldsymbol{h}}$ for the mediator. Such an operation is called an arbitration.

In the previous example, the possibility of exchange is of interest to two consumers - mediation is two-way. Multilateral mediation is also possible in cases where multiple consumers are involved in the exchange. A mediator that facilitates the transaction will be able to benefit from this. In the future, we assume that either the mediator is himself a participant in the economic process, or the charge $\boldsymbol{\omega}_{\boldsymbol{h}}$ for the mediation is small enough and can be neglected.

We will call a state, in which both bilateral and multilateral mediation are impossible, all operations have already been completed, no exchange can take place, a stable distribution Obviously, there are no reasons why this state must coincide with the competitive equilibrium.

The stable distribution $\boldsymbol{E}^{\mathbf{0}}$, defined in this way is obviously the optimum of distribution. Otherwise, there would be another possible condition $E^{l}$, selected by a random consumer that others consider to be no worse than $\boldsymbol{E}^{\mathbf{0}}$. The statement that $E^{1}$ is possible is tantamount to the statement that the transition from $\boldsymbol{E}^{\mathbf{0}}$ to $E^{l}$ is an exchange.

Therefore, there is a possibility of mediation (which may cover all consumers), which is contrary to the stability of distribution $\boldsymbol{E}^{\mathbf{0}}$. The concept of mediation can also be used to describe the exchange process. If the initial position in which each consumer owns $\boldsymbol{\omega}_{\boldsymbol{i} h}$, is not a stable distribution, some exchanges and mediation may occur. The amounts of goods owned by different participants change as many times as necessary for a stable distribution. The benefits of $\boldsymbol{S}_{\boldsymbol{i}}$ cannot be diminished during these exchanges. Assuming that no advantageous opportunity remains missed (that is, the information is fairly well disseminated to the mediator, or that no participant refuses the advantageous for him operation if he could behave having formulated the requirements acceptable to others), then such a process converges.

The disadvantage of such a theory is that there may be different ways to achieve a stable distribution. 


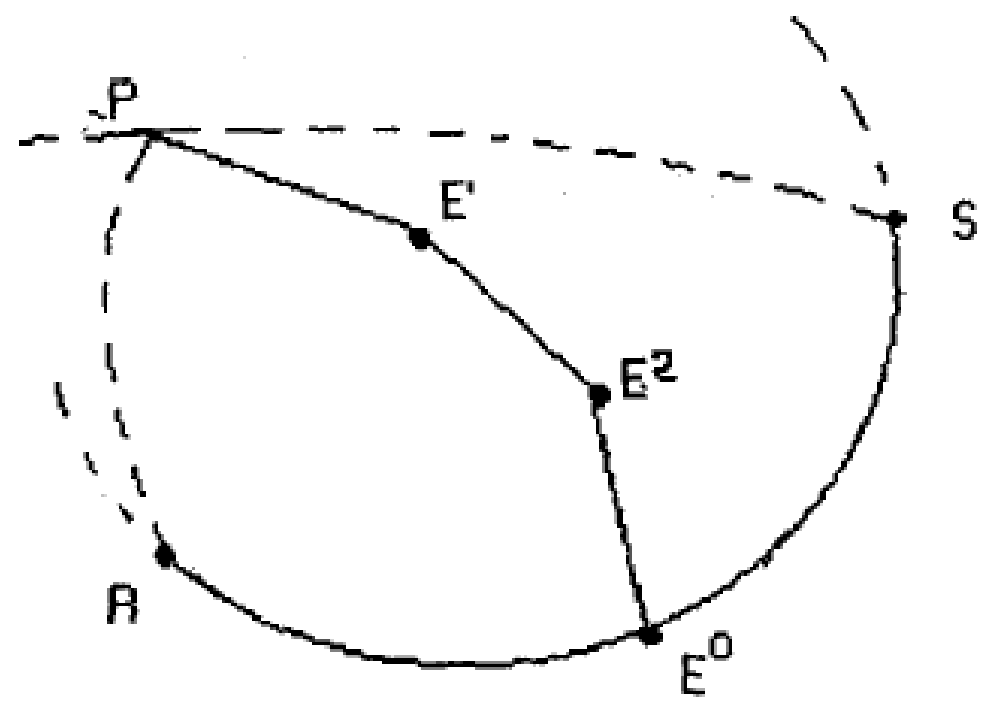

Fig. 4

Let us explain this in the example of the Edgeworth diagram for obtaining the two goods and two participants shown in Fig. 4. Curves $P R$ and $R S$ are indifference curves passing through the point $P$ of the initial stock. The RS curve is the geometric location of the Pareto optimum. The path consisting of three exchanges (from $P$ and $E^{l}$, from $E^{l}$ and $E^{2}$, from $E_{2}$ to $E^{0}$, depicted by a polygonal chain $\boldsymbol{P} \boldsymbol{E}^{\mathbf{1}} \boldsymbol{E}^{\mathbf{2}} \boldsymbol{E}^{\mathbf{0}}$. Each exchange increases the satisfaction of both consumers. However, you can imagine many different paths ending at any point of the curved line $R S$.

\section{The Kernel in the Economy of Exchange}

the Economy of Exchange is inherently a game because, under some constraints, participants choose their own strategies, the combined action of which ultimately leads them to reach some utility levels $\boldsymbol{S}_{\boldsymbol{i}}$. It is difficult to describe the primary actions of the exchangers: worries, offers, counterproposals, etc. In the Economy of Exchange, the distributions are determined by the levels of utility corresponding to the consumption vectors. Now we can think directly on the basis of the set consisting of $t$ vectors $\boldsymbol{x}_{\boldsymbol{i}},-$. The general definitions that were given earlier can be easily transferred to this case.

The coalition is a subset of $C$ of the set $t$ of consumers.

State $\boldsymbol{E}^{\mathbf{0}}$ is possible for coalition $C$ if

$$
\boldsymbol{x}_{\boldsymbol{i}}^{\mathbf{0}} \in \boldsymbol{X}_{\boldsymbol{i}}, \boldsymbol{i} \in \boldsymbol{C}
$$




$$
\sum\left(x_{i h}{ }^{0}-\omega_{i h}\right)=0, h=1,2, \ldots, l .
$$

Conditions (48) and (49) ensure that the achievement of $\boldsymbol{x}_{\boldsymbol{i}}^{\mathbf{0}}$ is possible for Coalition $\mathrm{C}$ members acting jointly and independently of other noncoalition members. State $\boldsymbol{E}^{\mathbf{0}}$ is possible if it is possible for a coalition of all members. $\boldsymbol{E}^{\mathbf{0}}$ is blocked by Coalition $\mathrm{C}$, if there is a state $E^{l}$, possible for C such as

$$
S_{i}\left(x_{i}^{1}\right) \geq S_{i}\left(x_{i}^{0}\right), i \in C,
$$

with strict inequation for at least one member of C.Condition (50) guarantees that $\boldsymbol{x}_{\boldsymbol{i}}{ }^{\mathbf{1}}$ is preferable to $\boldsymbol{x}_{\boldsymbol{i}}{ }^{\mathbf{0}}$ for members of $\mathrm{C}$. The kernel of the economy of exchange is obviously a set of possible states $E$, not blocked by any coalition. It is contained in the set of all the optimum distribution, but contains all the competitive equilibria. Let us graphically represent a kernel for the case where there are two goods and two consumers (Fig. 5), constructed on the basis of the Edgeworth diagram.

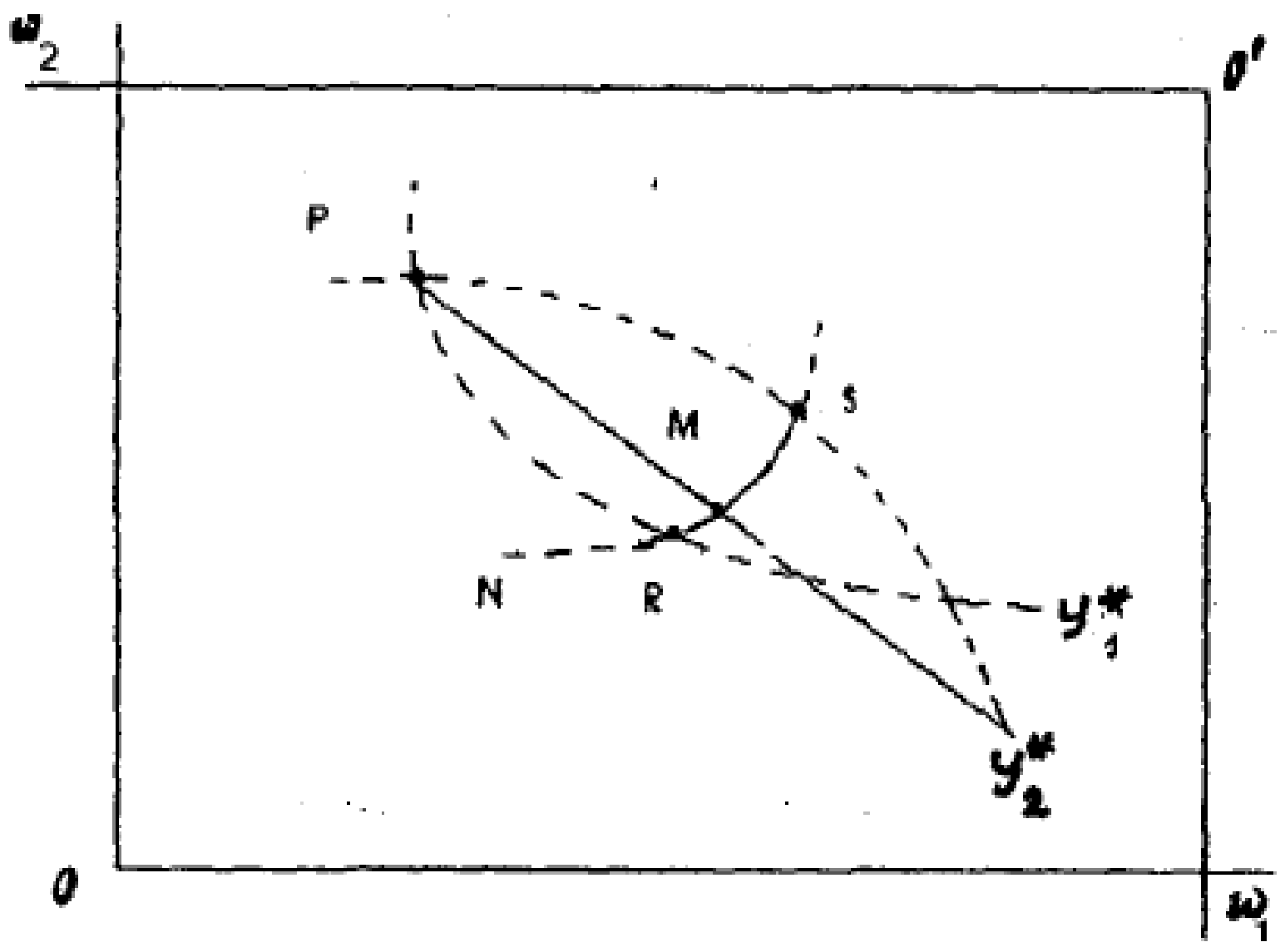

Fig. 5 
It is known that the kernel is represented by a portion of the $M N$ curve, which is the geometric location of the distribution optimum, that is, the points at which the indifference curves of two consumers touch each other. The states depicted by external points with respect to the $M N$ points blocked by the coalition $\{1,2\}$. In addition, states blocked by Coalition $\{1\}$ are the points located to the left of the indifference curve $\boldsymbol{y}_{\mathbf{1}}{ }^{*}$ passing through the point $P$, which is the initial distribution of resources between consumers. The states blocked by coalition $\{2\}$ are those points that are located to the right of the indifference curve $\boldsymbol{y}_{2}{ }^{*}$, passing through $P$. Thus, the kernel is a part of the curve $M N$, extending from the intersection point from $\boldsymbol{y}_{1}{ }^{*}$ to point of intersection with $\boldsymbol{y}_{2}{ }^{*}$. We see that the competitive equilibrium is $M$, where the common tangent to both indifference curves passes through $P$, belongs to the kernel. In the graph (see Fig. 5), the set of states of stable distribution coincides with the kernel everywhere except the boundary points Rand $S$.. An arbitrary non-kernel distribution determines the state in which mediation is possible. Conversely, an arbitrary kernel-owned state $\boldsymbol{E}^{\mathbf{0}}$ (except $R$ and $S$ ) is a stable distribution for the specified economy, since the transition from the initial state $P$ to the state $\boldsymbol{E}^{\mathbf{0}}$ is made through favorable mediation and no mediation is possible, after $\boldsymbol{E}^{\mathbf{0}}$ is reached. This attribute does not take place if there are more than two participants. The reason for this is the difference of opinion on the equilibrium establishment process.

Let us suppose there are two goods and three participants who initially own the resources in quantities

$$
\omega_{1}=\left(\begin{array}{l}
0 \\
2
\end{array}\right), \omega_{2}=\left(\begin{array}{l}
1 \\
1
\end{array}\right), \omega_{3}=\left(\begin{array}{l}
1 \\
1
\end{array}\right) .
$$

We believe that the benefits of these consumers are the same and are described by the following utility functions:

$$
S_{i}\left(x_{i}\right)=x_{i 1} x_{i 2}, x_{i h} \geq 0 .
$$

The following two exchanges determine the possible path that ends in a sustainable distribution. Consumers 1 and 2 enter into an agreement that the former gives the latter $3 / 2$ of good 2 in exchange for $1 / 4$ of good 1 . The pleasure of the first increases from 0 to $1 / 8$, the pleasure of the second from 1 to 15/8. After sharing, everyone has the following goods:

$$
x_{1}=\left(\begin{array}{l}
1 / 4 \\
1 / 2
\end{array}\right), x_{2}=\left(\begin{array}{l}
3 / 4 \\
5 / 2
\end{array}\right), x_{3}=\left(\begin{array}{l}
1 \\
1
\end{array}\right)
$$


Then the second and third parties enter into an agreement whereby the third party gives the second the $1 / 4$ of the good 1 in exchange for the $1 / 2$ of the good 2 . The pleasure of the second increases from 15/8 to 2 , the pleasure of the third - from 1 to 9/8. Ultimately, consumers will own the goods

$$
x_{1}^{0}=\left(\begin{array}{l}
1 / 4 \\
1 / 2
\end{array}\right), x_{2}^{0}=\left(\begin{array}{l}
1 \\
2
\end{array}\right), x_{3}^{0}=\left(\begin{array}{l}
3 / 4 \\
3 / 2
\end{array}\right)
$$

It is easy to check that the obtained state $\boldsymbol{E}^{\mathbf{0}}$ is a stable distribution; this is the optimal distribution to which prices $p_{1}=2, \mathrm{p}_{2}=1$ can be linked.

According to the definitions we adhere to, the state does not belong to the kernel because it is blocked by a coalition consisting of the first and third party.

By pooling their initial resources defined by vectors (51), they could distribute

$$
x_{1}^{*}=\left(\begin{array}{c}
1 / 4 \\
1
\end{array}\right), x_{3}^{*}=\left(\begin{array}{c}
3 / 4 \\
2
\end{array}\right)
$$

which, for them, is obviously better than the distribution shown by vectors (54). As this example shows, the difference between the kernel and the set of stable distributions does not lie in the difference of approaches which use the central concepts of «arbitration» and «coalition», respectively.

Arbitration can be defined as an operation whereby a coalition moves from one division to another, which is best for its members. The difference lies in the description of the exchange implementation process.

The idea that the final distribution must belong to the kernel does in fact implicitly imply non-kernel agreements, which could lead to the nonkernel results, or similar agreements that have already been concluded and may be terminated for the sake of others. To clarify this idea, Edgeworth hypothesized that parties could freely renegotiate agreements, that is, concluded contracts could always be canceled later, if a better contract is possible.

The hypothesis that contracts are not considered conclusive before the state inside the kernel is reached is very unrealistic. In addition, it should not be taken literally. Rather, it means that the participants do not make the final decisions before they evaluate the outcome of the various possible contracts. 
The ability to renegotiate contracts accepted by Edgeworth is essentially similar to the Walras hypothesisaccording to which the contracts are not concluded until the equilibrium prices are established. It implies that there is a great deal of opportunity for contracts between the parties and leads to a fairly accurate theory.

Rejecting this possibility, the stable distributions obtained from a given initial situation are very uncertain, especially in economies with a large number of participants. Of course, we know that this distribution will be optimum and that it is more preferable than the initial situation for all participants. But with the help of general logical analysis nothing more definite can be said. We have to choose between two theories: a less restrictive but less accurate theory of stable distribution, and a more restrictive but more accurate theory of kernel.

Again, if the number of participants in the exchange is large, then the costs of information and communication can significantly complicate finding the distribution belonging to the kernel To accept that the end result lies within the kernel means to assume that the optimality problem, which is the subject of a great part of microeconomic theory, is solved.

\section{Closed Bid Simulation}

In closed tenders, tenderers tend to announce their bids, usually once, without informing each other. The lowest or highest bid is accepted depending on the type of bidding.

An example of the first case may be a competition for the cheapest project of an administrative building, an example of the second case is the rent offer for the right to use the parking lot by the firms.

Before deciding on bidding and setting a bid, it is necessary to estimate the costs associated with the object of the auction. Typically, a rate that exceeds these costs is set, and if accepted, the difference is the amount of profit.

In the case of closed bidding, only the winning bid is often announced. For certainty, we will consider the case when the lowest bid is accepted. Then we can have cost estimates and minimum rates for cases when contracts are not concluded. Let us suppose that based on the accumulated statistics of the ratio

$$
\mathrm{x}=\text { Lowest Bid / Cost Estimate }
$$


has a normal distribution with mathematical expectation $\mathrm{c}$ and a variance of $\boldsymbol{\delta}^{\mathbf{2}}$. The task of bidders is to set a bid that maximizes expected profit.

Let own costs according the certain contract be equal to $c$ and the tenderer has set a price of $p$. Then its profit is $p-c$, when this price was the lowest, and equals to 0 otherwise.

The probability that this participant has set the lowest price is equal to the probability that the ratio $p / c$ will be less than a random variable that has a normal distribution with a mathematical expectation ц and a variance $\boldsymbol{\delta}^{\mathbf{2}}$. This probability is equal to $(\boldsymbol{p} / \boldsymbol{c})$, where

$$
f(z)=\frac{1}{\sqrt{2 \pi \delta}} \int_{z}^{\infty} e^{-\frac{(x-\mu)^{2}}{2 \delta^{2}}} d x .
$$

Therefore, the expected profit is

$$
\boldsymbol{P}=(\boldsymbol{p}-\boldsymbol{c}) \boldsymbol{f}\left(\frac{\boldsymbol{p}}{\boldsymbol{c}}\right) .
$$

We need to maximize $P$ by, If we find the first derivative of the last expression by $p$ and equate it to zero, we obtain

$$
\frac{d P}{d p}=f\left(\frac{p}{c}\right)+(p-c) f^{\prime}\left(\frac{p}{c}\right) \frac{1}{c}=0 .
$$

We denote $\frac{p}{c}=\boldsymbol{z}$, then

$$
f(z)+(z-1) f^{\prime}(z)=0 .
$$

If we put $=\boldsymbol{\mu}+\boldsymbol{t} \boldsymbol{\delta}$, then

$$
f(z)=\frac{1}{\sqrt{2 \pi}} \int_{t}^{\infty} e^{-\frac{x^{2}}{2}} d x, f^{\prime}(z)=-\frac{1}{\sqrt{2 \pi \delta}} e^{-\frac{t^{2}}{2}},
$$

and to find $t$ it is necessary to solve the equation

$$
\frac{1}{\sqrt{2 \pi}} \int_{t}^{\infty} e^{\frac{x^{2}}{2}} d x=(\mu+t \delta-1) \frac{1}{\sqrt{2 \pi \delta}} e^{\frac{t^{2}}{2}}
$$

The values of the integral and the exponents can be found in the tables of normal distribution and the equation can be solved by the approximate (graphical) method (Fig. 6): 


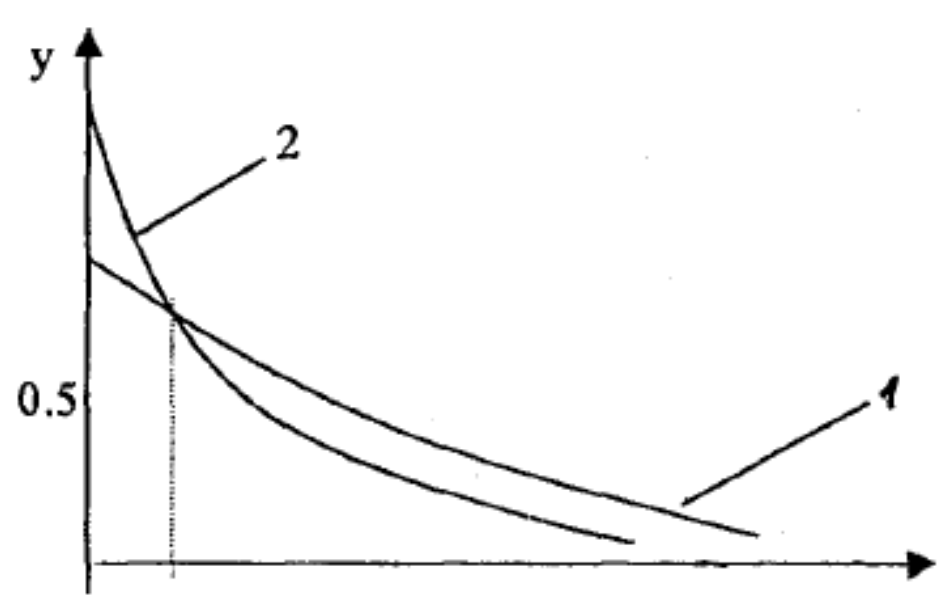

$0 \quad t_{0}$

$\mathbf{t}$

Fig. 6

where the curves 1 and 2 are the graphs of the functions $y_{1}(t)$ and $y_{2}(t)$, which are respectively in the left and right parts of the equation. If the solution of the last equation, then the optimal rate that maximizes expected profit,

$$
p_{0}=c\left(\mu+t_{0} \delta\right)
$$

The foregoing calculations implicitly assumed that the winning bid distributions did not change with any change in the behavior of one of the bidders. Such an assumption holds for a short period of time when a large number of independently operating firms are involved in the auction.

\section{Modeling of auction bidding}

Let $\mathrm{u}$ suppose that two objects are offered for sale one by one at the auction, and there are two buyers $A$ and $B$, own $\$ 100$ and $\$ 140$ respectively. It is assumed that the known prices at which the purchased objects can then be sold: the first object is priced at $\$ 75$, the second is $\$ 125$. Consider the problem of defining buyer strategies to maximize their profits ${ }^{2}$.

If an item of value of $\$ 75$ is initially offered for sale, then the buyer $A$ will raise the bid as long as its profit in the case of acquisition of the item for $\$ 75$ is not equal to its profit in the case of purchase of the object for $\$ 125$. If $B$ is able to buy an object worth $\$ 75$ for $\$ x$, then he will have

\footnotetext{
${ }^{2}$ Akof R., Sasiena M. Fundamentals of Operations Research. Moskow: World, 2007, 2010 p.
} 
$\$ 140-\$ x$ and Ashould purchase an object worth $\$ 125$ for an amount that is a bit higher than the $140-\mathrm{x}$ provided $140-\boldsymbol{x} \leq \mathbf{1 0 0}$.

If $A$ acquires the first object for $\$ \mathrm{x}$, then his profit will be $\$ 75-\mathrm{x}$, and if the first object at the same price is acquired by $B$, then the profit $A$ will be equal to $125-(140-\mathrm{x})$. Therefore $A$ will raise the price until the condition is fulfilled

$$
75-x=125-(140-x)=x-15,
$$

whence $x=45$.

The buyer $B$ understands that he could purchase both objects. If he can buy the first object for less than $\$ 40$, then he will for sure buy the second for a price of just over $\$ 100$. Thus, if $B$ is able to get the first object for the sum of $y<40$, then its total profit will be

$$
75-y+125-100=100-y .
$$

However, if the first object is for $A$ the sum $y$, then $B$ will receive the second one for the sum $\$ 100-y$, and therefore, its profit will be equal

$$
125-(100-\mathrm{y})=25+y \text {. }
$$

As a result, $B$ will raise the price for the first item until the condition is fulfilled

$$
100-y=25+\mathrm{y}
$$

whence $y=37.5$.

Assuming that both participants of the auction made these calculations, then the buyer $B$ will reach the following conclusions: the maximum price he can offer for the first object, when he intends to pay for both, is $\$ 37.5$; the buyer $A$ will never allow him $(B)$ to acquire the first object for less than $\$ 45$, as otherwise the profit for $A$ will be less than he can afford if he raises the bid to this price; if $A$ acquires the first object, the more he pays for it, the cheaper the second item will be for $B$ buy it.

It follows that the buyer $B$ will force $A$ to pay for the first object of $\$ 45$, and the second one will be sold to $B$ for the price of $\$ 100-\$ 45=\$ 55$. The profit of $A$ will be $\$ 75-\$ 45=30 \$$, and the profit of $B$ will be $\$ 125-\$ 55=\$ 70$.

Note that when the number of items being auctioned exceeds two, the approach of finding the best strategies for buyers $A$ and $B$ becomes very cumbersome and practically impossible to put into practice. In this case, it 
is appropriate to consider such a problem as a dynamic programming problem and solve it in stages.

Let us suppose that only one object is put up for auction and Aowns the sum $\boldsymbol{\alpha}$, a $B$ has the sum $\boldsymbol{\beta}$. Both participants of the auction consider that the value of the object is $\boldsymbol{c}_{\mathbf{1}}$.

Let us denote by $\boldsymbol{u}_{\mathbf{1}}(\boldsymbol{\alpha}, \boldsymbol{\beta})$ the profit of $A$, and by $\boldsymbol{v}_{\mathbf{1}}(\boldsymbol{\alpha}, \boldsymbol{\beta})$ - the profit of $B$.

If $B$ has set the price $x$, then $A$, having increased it to the value $+\Delta$, will purchase the object and receive a profit $\boldsymbol{c}_{\mathbf{1}}-\boldsymbol{x}-\Delta$. If the object is purchased by $B$, then $A$ will not receive any profit. Therefore $A$ will increase the price provided that $\boldsymbol{x}<\boldsymbol{c}_{\mathbf{1}}$. In addition, since $A$ has only the sum a, condition $\boldsymbol{x}<\boldsymbol{\alpha}$ must be satisfied. Obviously, $B$ will think similarly. The following conclusions can be drawn from here:

1) if $\boldsymbol{\alpha}>\boldsymbol{\beta}$ and $\boldsymbol{\beta}>\boldsymbol{c}_{\mathbf{1}}$, then the object will be purchased by $A$ at a price that slightly exceeds $\beta$ and $\boldsymbol{u}_{\mathbf{1}}(\alpha, \beta)=\boldsymbol{c}_{\mathbf{1}}-\boldsymbol{\beta}$, and $\boldsymbol{v}_{\mathbf{1}}(\alpha, \beta)=\mathbf{0}$;

2) if $\boldsymbol{\alpha} \geq \boldsymbol{c}_{\mathbf{1}}$ and $\boldsymbol{\beta} \geq \boldsymbol{c}_{\mathbf{1}}$, then the object will be purchased at the price of $c_{1}$ and $u_{1}(\alpha, \beta)=v_{1}(\alpha, \beta)=0$;

3) if $\boldsymbol{\alpha}<\boldsymbol{\beta}$ and $\boldsymbol{\alpha}<\mathrm{c}_{\mathbf{1}}$, , then the object will be purchased by $A$ at a price that slightly exceeds $\alpha$ and $\boldsymbol{u}_{\mathbf{1}}(\alpha, \beta)=\mathbf{0}$, and $\boldsymbol{v}_{\mathbf{1}}(\boldsymbol{\alpha}, \boldsymbol{\beta})=\mathrm{c}_{\mathbf{1}}-\boldsymbol{\alpha}$.

Now let us suppose that the second object is being auctioned with value $c_{2}$, and it is being offered first. We denote by $\boldsymbol{u}_{\mathbf{2}}(\boldsymbol{\alpha}, \boldsymbol{\beta})$ and $\boldsymbol{v}_{2}(\boldsymbol{\alpha}, \boldsymbol{\beta})$ the total profits of $A$ and $B$, when two objects are auctioned.

If $B$ has set the price $\mathrm{x}$ for $\mathrm{c}_{2}$, then $A$ may give him a chance to purchase this object and the total profit of $A$ will be $\boldsymbol{u}_{\mathbf{1}}(\boldsymbol{\alpha}, \boldsymbol{\beta}-\boldsymbol{x})$. However, $A$ may raise the price a little more than $x$, in this case when $B$ gives way to it, the profit $A$ will be equal to $c_{2}-x+u_{1}(\alpha-x, \beta)$.

If $A$ has enough resources, then he will continue to raise the price until conditions are met

$$
x \leq \alpha, c_{2}-x+u_{1}(\alpha-x, \beta) \geq u_{1}(\alpha, \beta-x)
$$

Similarly $B$ will raise its rates as long as the conditions are met

$$
x \leq \beta, c_{2}-x+v_{1}(\alpha, \beta-x) \geq v_{1}(\alpha-x, \beta)
$$

If you tabulate the values of the functions $\boldsymbol{u}_{\mathbf{1}}(\alpha, \beta)$ and $v_{\mathbf{1}}(\alpha, \beta)$ at different values of $\boldsymbol{\alpha}, \boldsymbol{\beta}$, it is easy to find the smallest values $x$ under which these conditions begin to be violated. Let them respectively be equal to $\boldsymbol{x}_{1}{ }^{(2)}$ and $\boldsymbol{x}_{2}{ }^{(2)}$. Then we conclude that with respect to object $\mathrm{c}$ the 
participant $A$ of the auction will increase bids up to $\min \left(x_{1}{ }^{(2)}, \alpha\right)$, and $B$ - up to $\min \left(\boldsymbol{x}_{\mathbf{2}}{ }^{(2)}, \boldsymbol{\beta}\right)$. An object $\mathrm{c}_{2}$ will be bought by $A$ provided

$$
\min \left(x_{1}{ }^{(2)}, \alpha\right)>\min \left(x_{2}{ }^{(2)}, \beta\right)
$$

When we know who acquired the object $c_{2}$, we define (tabulate) the functions $\boldsymbol{u}_{\mathbf{2}}(\boldsymbol{\alpha}, \boldsymbol{\beta})$ and $\boldsymbol{v}_{\mathbf{2}}(\boldsymbol{\alpha}, \boldsymbol{\beta})$.

Now let us suppose that the other object is being auctioned with value $\boldsymbol{c}_{\boldsymbol{i}}$, and it is being offered first. Then $A$ and $B$ will raise prices as long as the conditions are met

$$
x \leq \alpha, c_{i}-x+u_{i-1}(\alpha-x, \beta) \geq u_{i-1}(\alpha, \beta-x)
$$

for $A$ and

$$
x \leq \beta, c_{i}-x+v_{i-1}(\alpha, \beta-x) \geq v_{i-1}(\alpha-x, \beta)
$$

for $B$.

If

$$
\min \left(x_{1}{ }^{(i)}, \alpha\right)>\min \left(x_{2}{ }^{(i)}, \beta\right),
$$

then the object $\boldsymbol{c}_{\boldsymbol{i}}$ will be acquired by $A$, otherwise it will go to $B$. Determining who purchases the object $\boldsymbol{c}_{\boldsymbol{i}}$, we find (tabulate) the functions $\boldsymbol{u}_{\boldsymbol{i}}(\boldsymbol{\alpha}, \boldsymbol{\beta})$ and $\boldsymbol{v}_{\boldsymbol{i}}(\boldsymbol{\alpha}, \boldsymbol{\beta})$ - the total profits of $A$ and $B$, when $i$ objects were auctioned.

Let $n$ items be auctioned. Then sequentially giving to $i$ the values 2 , $3, \ldots, n$, we get to the object $c_{n}$, which is put up for auction first. Analyzing the ratio

$$
\begin{aligned}
& x \leq \alpha, c_{n}-x+u_{n-1}(\alpha-x, \beta) \geq u_{n-1}(\alpha, \beta-x) ; \\
& x \leq \beta, c_{n}-x+v_{n-1}(\alpha, \beta-x) \geq v_{n-1}(\alpha-x, \beta),
\end{aligned}
$$

as noted above, depending on whichever is greater $\min \left(\boldsymbol{x}_{1}{ }^{(n)}, \boldsymbol{\alpha}\right)$ or $\min \left(\boldsymbol{x}_{2}{ }^{(\boldsymbol{n})}, \boldsymbol{\beta}\right)$, we determine who buys the object $c_{n}$, and the total profits $\boldsymbol{u}_{\boldsymbol{n}}(\boldsymbol{\alpha}, \boldsymbol{\beta})$ and $\boldsymbol{v}_{\boldsymbol{n}}(\boldsymbol{\alpha}, \boldsymbol{\beta})$ of the buyers $A$ and $B$. . Putting $\boldsymbol{i}=\boldsymbol{n}-\mathbf{1}, \boldsymbol{n}-\mathbf{2}, \ldots, 2$, we find who buys $\boldsymbol{c}_{n-1}, \boldsymbol{c}_{n-2}, \ldots, \boldsymbol{c}_{\mathbf{1}}$ objects and profits from each.

Let's solve the above-mentioned problem with the method of dynamic programming, when three objects worth $\boldsymbol{c}_{\mathbf{1}}=\mathbf{1 2 5} \boldsymbol{y} . \boldsymbol{o .}, \boldsymbol{c}_{\mathbf{2}}=\mathbf{7 5} \boldsymbol{y} . \boldsymbol{o}$., $c_{3}=3-0$ y.o. $\mathrm{m}$ are auctioned and buyers $A$ and $B$ have $\boldsymbol{\alpha}=\mathbf{1 0 0} \boldsymbol{y} . \boldsymbol{o}$. and $\boldsymbol{\beta}=140$ y.o. at their disposal 
If one object $\boldsymbol{c}_{\mathbf{1}}$ is being auctioned, then it will be purchased by $B$ at a price slightly higher than $\boldsymbol{u}_{\mathbf{1}}(\alpha, \beta)=\mathbf{0}, \boldsymbol{v}_{\mathbf{1}}(\alpha, \beta)=125-100=$ $=25$ y.o.

Now let us suppose that two objects $\boldsymbol{c}_{\mathbf{1}}$ and $\boldsymbol{c}_{\mathbf{2}}$ are being put up for auction, and $c_{2}$ is being offered first. Let us determine who acquires object $c_{2}$ this time, and who $-\boldsymbol{c}_{\mathbf{1}}$ at different values of $\alpha \leq \mathbf{1 0 0}$ and $\boldsymbol{\beta} \leq \mathbf{1 4 0}$, using the relation $(21)-(23)$.

Let us consider the case when $\boldsymbol{\alpha}<\boldsymbol{\beta}$ ( $\boldsymbol{\beta}>\boldsymbol{\alpha}$ is symmetrical, buyers $A$ and $B$ change places).

Then

$$
\begin{gathered}
u_{1}(\alpha-x, \beta)=0, u_{1}(\alpha, \beta-x)=\left\{\begin{array}{c}
0, \text { коли } x<\beta-\alpha, \\
125-(\alpha-x), \text { коли } x>\beta-\alpha ;
\end{array}\right. \\
v_{1}(\alpha, \beta-x)=\left\{\begin{array}{c}
125-\alpha, \text { коли } x<\beta-\alpha, \\
0, \text { коли } x>\beta-\alpha ;
\end{array}\right. \\
v_{1}(\alpha-x, \beta)=125-(\alpha-x)
\end{gathered}
$$

and conditions (55), (56) have the form

$$
\begin{aligned}
& \left\{\begin{array}{c}
75 \geq x \\
\alpha \geq x,\left\{\begin{array}{c}
37,5 \geq x, \\
\beta \geq x,
\end{array} \text { коли } x<\beta-\alpha,\right.
\end{array}\right. \\
& \left\{\begin{array} { c } 
{ \frac { \beta - 5 0 } { 2 } \geq x , } \\
{ \alpha \geq x , }
\end{array} \left\{\begin{array}{c}
\frac{\alpha-50}{2} \geq x, \\
\beta \geq x, \text { коли } x>\beta-\alpha .
\end{array}\right.\right.
\end{aligned}
$$

Put in them $\alpha=100, \beta=140$. Then from (58) it follows

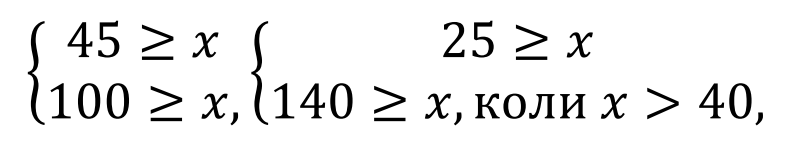

and $x_{1}{ }^{(2)}=45$,

Since

$$
\begin{gathered}
\min \left(x_{1}{ }^{(2)}, \alpha\right)=\min (45,100)=45, \\
\min \left(x_{1}{ }^{(1)}, \beta\right)=\min (37,5,140)=37,5,
\end{gathered}
$$

then, under condition (57) (45>37.5), object $c_{2}$ is acquired by the buyer A at a price of $\$ 45$. and his profit is $u_{2}(100,140)=75-45=30$ y. $o$. 
Buyer B will purchase the object $c_{1}$ at a price of $\$ 100-\$ 45=\$ 55$. and his profit will be $v_{2}(100,140)=125-55=70$ y. $o$.

This solution is the same as the two-object solution obtained above by the second method.

Using conditions (58), (59), similar to the above, we determine who acquires object $c_{2}$ at $\alpha \leq 100, \beta \leq 140$. Knowing this, we calculate (tabulate) functions (profits) $u_{2}(\alpha, \beta), v_{2}(\alpha, \beta)$ (Table 1).

Table 1

\begin{tabular}{|c|c|c|c|c|}
\hline \multirow{2}{*}{$\beta$} & \multicolumn{4}{|c|}{$\alpha$} \\
\cline { 2 - 5 } & 100 & 90 & 86 & 85 \\
\hline 140 & 30 & 25 & 21 & 20 \\
& 70 & 85 & 93 & 95 \\
\hline 130 & 35 & 35 & 31 & 30 \\
& 65 & 75 & 83 & 85 \\
\hline 126 & 38 & 37 & 35 & 25 \\
& 62 & 73 & 79 & 105 \\
\hline 125 & 37,5 & 37,5 & 36 & 35 \\
& 62,5 & 72,5 & 78 & 80 \\
\hline
\end{tabular}

In the table, the top row is the value of function $\boldsymbol{u}_{\mathbf{2}}(\boldsymbol{\alpha}, \boldsymbol{\beta})$, the bottom row is the value of function $\boldsymbol{v}_{2}(\boldsymbol{\alpha}, \boldsymbol{\beta})$.

Now let us suppose that the third object is being auctioned with value $c_{3}=30$ y.o., and it is being offered first. Then A and B will raise prices as long as the conditions are met

$$
\begin{aligned}
& x \leq 100,30-x+u_{2}(100-x, 140) \geq u_{2}(100,140-x) ; \\
& x \leq 140,30-x+v_{2}(100,140-x) \geq v_{2}(100-x, 140) .
\end{aligned}
$$

Using the table 19 , we easily find that when $x=10$

$30-10+25>35$

$30-10+62=85$,

that is, $\boldsymbol{x}_{\mathbf{2}}{ }^{(\mathbf{3})}=\mathbf{1 0}$, and when $x=14$

$30-14+21 \approx 38$;

$30-14+62<93$, 
that is $x_{1}{ }^{(3)} \approx 14$ Since $x_{1}{ }^{(3)}>x_{2}{ }^{(3)}$, the object $c_{3}$ will be bought by the buyer $A$ at the price of $\$ 14$. and his profit will be $30-14=\$ 16$.

After that, he has $\$ 100-\$ 14=\$ 86$, and buyer $B$ has $\$ 140$. Using the table 19 for these values we find the profits from the other two objects $\boldsymbol{c}_{\mathbf{2}}$ and $\boldsymbol{c}_{\mathbf{1}}$. Accordingly, they are $\$ 21$ for $A$ and $\$ 93$ for $B$, and $A$ acquires object $c_{2}$ at the price of $\$ 54$. (profit $\$ 75-\$ 54=\$ 21$ ), and $B$ will get the object $c_{x}$ at a price of $\$ 32$.

(profit $\$ 125-\$ 32=\$ 93$ ).

Therefore, when three objects are auctioned at a cost of $c_{1}=125 y . o ., c_{2}=75 y . o . c_{3}=30 y . o$. , the buyer $A$, in order to maximize his profit, will buy the objects $\boldsymbol{c}_{\mathbf{3}}$ and $\boldsymbol{c}_{\mathbf{2}}$ and his total profit will be $\$ 16+\$ 21=\$ 37$, and the buyer $B$ will buy the object $\boldsymbol{c}_{\mathbf{1}}$ (the profit is $\$ 93$ ).

\section{REFERENCES}

1. Malenvo E. Lectures on Microeconomic Analysis. Moskow: Science, 1985, $392 \mathrm{p}$.

2. Akof R., Sasiena M. Fundamentals of Operations Research. Moskow: World, 2007, 2010 p.

Information about the author: Medvediev M. H.

Doctor of Technical Sciences, Professor, Head at the General Engineering and Thermal Power Engineering Department of the V. I. Vernadsky Taurida National University 